\title{
Spectral Differences Between Normal and Atherosclerotic Aorta
}

\author{
Nikolay Korovin*
}

\begin{abstract}
Ischemic heart disease associated with coronary artery atherosclerosis is a leading cause of death in the world today. In addition to standard treatments such as balloon angioplasty, laser mediated angioplasty is being considered as a potential adjuvant or replacement. Nevertheless, experiments and clinical experience have demonstrated that laser angioplasty is associated with damage to normal vessel tissue, which can cause serious complications. To study the possibility of minimizing these effects by directing laser energy more specifically to atherosclerotic lesions, data concerning the spectral characteristics of normal and diseased artery are necessary. In the current study, the absorbance, reflection and fluorescence spectra of normal and atherosclerotic aortic wall tissue are defined, revealing that (i) spectral characteristics of atherosclerotic aorta wall samples are significantly differed from that of healthy vascular wall samples and (ii) based on a spectral analysis of vascular wall, it is possible to distinguish morphological types of atherosclerotic plaques (i.e., lipidic, calcified). The current study contributes to a more complete understanding of laser-tissue interactions that may, following more experimentation and technique development, result in an improvement of clinical laser angioplasty technique.
\end{abstract}

\section{INTRODUCTION}

Atherosclerotic disease is a leading cause of death in the world today, and lesions of both coronary vessels and peripheral arteries are associated with increased morbidity and mortality (1). The occlusion of coronary vessels by atherosclerotic lesions typically results in ischemic heart disease. Considering its widespread prevalence, as well as its associated morbidity and mortality, effective treatment of atherosclerosis will be of great benefit to a vast number of patients. One example of a minimally traumatic intervention currently used to reduce atherosclerosis-induced stenosis is balloon dilatation of blood vessels (1). Unfortunately, despite the success of this technique, balloon angioplasty is not well suited for some complex lesion types, including total occlusion. Alternative minimally

\footnotetext{
* To whom correspondence should be addressed: Department of Cardiovascular Surgery, Institute of Cardiology, Tomsk 111A, Kievskaya St., Tomsk, Russia 634012; kvn@cardio.tsu.ru
}

invasive modalities for reducing coronary atherosclerotic lesions should prove very important in the future as adjuvants or replacements to balloon angioplasty. One promising method is the use of high power laser light technology to ablate atherosclerotic lesions (2-5).

In the 1980s, various laser types were evaluated for their efficacy and safety for use in coronary angioplasty. Argon $(514 \mathrm{~nm}$ ) and Nd:YAG (neodymium:yttriumaluminum-garnet) $(1060 \mathrm{~nm})$ continuous wave laser were two of the first to be evaluated clinically (6-8). These lasers destroy atherosclerotic lesions primarily via non-specific thermal action; in essence they burn the lesion. Unfortunately, both argon and Nd:YAG continuous wave induced considerable thermal damage to surrounding vessel tissue $(9,10)$.

An alternative laser technology used for the ablation of obstructive coronary lesions is the excimer laser (10-13). Its active medium is composed of rare gas halides (binary halogens) that emit light in the ultra-violet 
spectrum. The laser-light energy forces electrons from atoms, splitting chemical bonds. Thus the atherosclerotic lesion is destroyed in a photoablative rather than a thermal fashion. Fortunately, ultra-violet excimer lasers have proved more effective at clearing coronary lesions than other lasers evaluated, and are associated with little corollary thermal damage (10-13). Excimer laser removal of coronary atherosclerotic lesions has generated positive clinical and angiographic effects in patients. Notably, improvements have been recorded in patients with complex lesions (e.g., long, ostial, or total lesions) who receive excimer laser angioplasty as compared to traditional balloon angioplasty (14-23). Other studies, however, report no change, or even worse clinical outcome for patients receiving excimer laser versus balloon angioplasty for certain lesions (e.g., more than $10 \mathrm{~mm}$ or in vessels with a reference diameter of more than $2.5 \mathrm{~mm}$ ) (24-26). As such, a more complete understanding of excimer laser performance is necessary to determine the clinical circumstances in which it will be most effective.

Despite the potential for improved patient outcomes with the use of excimer laser angioplasty, various report have indicated serious complications, most of which are related to the damage of neighboring, healthy vessel tissue. Notably, clinical trials and basic studies have uncovered the occurrence of tissue tears under the influence of shock waves (consisting of gaseous bubbles) that arise during laser illumination, which can lead to the development of serious complications during the postoperative period $(10,22,27,28)$. It has been observed that local vessel saline infusion can reduce the severity of these shock waves, and thereby limit damage. In general, however, clinical outcomes following laser angioplasty would likely be improved and complications minimized by increasing the specificity of the laser action, thus limit the effects on healthy tissue, while at the same time maximizing its influence on the lesion.

In order to improve the therapeutic angioplasty and to minimize adverse effects, data concerning the reflection and absorption of energy, as well as the fluorescence of molecular structures within atherosclerotic plaques and normal tissue are essential (29-33). Spectral criteria for identification of vascular wall morphological types will help determine the optimal choice of laser energy and wavelength. Healthy, as well as primarily calcified, fibrous and fatty atherosclerotic lesions, considering their different molecular profiles, respond differently to laser light, and should not have exactly similar spectral characteristics. As such, healthy tissue and the various lesion types will have different wavelengths where an optimal ablative action is found. In general, by considering the reflection, absorption and fluorescence characteristics from healthy tissue and different lesion types, it will be possible to optimize conditions for lesion ablation and to minimize laser action and thus damage to normal vessel tissue.

In order to achieve this specificity, answers to two principal questions must be found. First, to attain the greatest lesion ablation, it is critical to determine in what region of the spectrum (specific wavelengths) atherosclerotic plaques exhibit maximal adsorption of laser light. Moreover, to minimize damage to healthy vessel tissue, it is necessary to determine the minimal adsorption wavelengths of the intact, healthy vessel. One way to approach this is a detailed experimental study of the spectral properties of atherosclerotic plaques and normal vessel wall tissue. Secondly, it must be determined whether there are optical characteristics of atherosclerotic plaques and of normal tissue that will permit them to be reliably distinguished. This would make it possible to establish preconditions for identification of a particular type of atherosclerotic lesion.

The present work has endeavored to define and evaluate several spectroscopic parameters of atherosclerotic and normal human aortic wall. To achieve this goal, the following tasks were undertaken: (i) to define the spectral parameters of healthy and atherosclerotic aortic wall; (ii) to compare the spectral characteristics of various types of atherosclerotic plaques with the spectral characteristics of the normal aortic wall; and (iii) to compare aortic wall morphology with data from spectral analysis. It is anticipated that this data will provide information that will be useful in the prediction of an optimal energy of laser for angioplasty and diagnosis of different atherosclerotic plaques morphological types. It must, however, be stressed that these data will need to be confirmed both in vivo and in coronary arteries before any definite clinical predictions can be made.

\section{METHODS}

The present work was conducted on 25 fragments of aortic arch from persons of both sexes (aged 50 to 80 years) who died of various causes. The experimental group consisted of samples of atherosclerotic aorta (lipidic, fibrotic and calcified plaques), while samples of normal aortic wall served as controls.

\section{Generation of the Absorbance Spectrum}

Cryostat sections of normal and lesioned aortic samples were used to record spectral absorbance in the ultraviolet range. Sections of 10 to $15 \mu \mathrm{m}$ thickness were placed between two pieces of quartz glass. Absorbance spectra were recorded with a SPh-26 scanning spectrometer, which measures absorbance spectrum in 


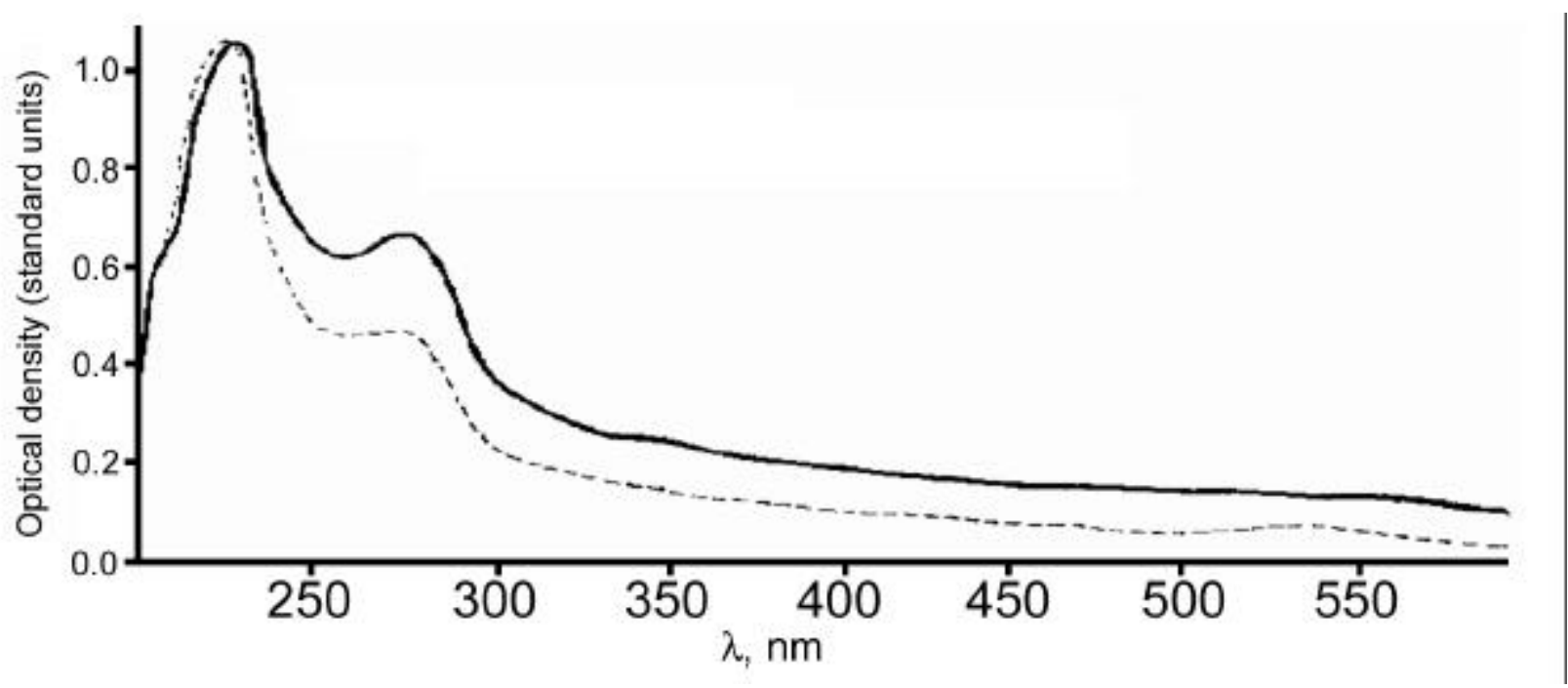

Figure 1A. The absorption spectra of normal aorta wall (solid line) and atherosclerotic plaque (dashed line) upon exposure to laser light in the ultraviolet range.

the range of 180 to $1100 \mathrm{~nm}$. A DVK-3 computer increased the sensitivity of the apparatus and enabled automated processing of the spectrophotometric signal with construction of a spectral absorption curve. The absorbance spectra were plotted as curves whose peaks correspond to the optical density of the substance.

\section{Recording of Reflection Spectra}

For recording of reflection spectra, preparations of atherosclerotic and normal aortic wall were used. The specimens were placed on a stand at an angle of $45^{\circ}$ to the direction of the beam of incident light. The excitation source was a Specol-10 with halogen lamp (retuning range $300-800 \mathrm{~nm}$ ); spectra were recorded with the aid of a spectrophotometer on a monochromator base with a diffraction grating (MDG-23) of resolution $6 \AA / \mathrm{mm}$. Light was focused onto the specimen to a section of $0.5 \times 0.5 \mathrm{~cm}$ through a micro-objective. With the aid of an achromatic condenser (focal distance $90 \mathrm{~mm}$ ), reflected light was directed onto the entry slit of the MDG-23 monochromator; the light was subsequently directed through the exit slit onto a photoelectron multiplier (PhEM-39). The signal from the PhEM was processed by an analognumerical transformer and the result was depicted graphically on the display screen of the DVK-3 computer. The graph depicted the dependence of intensity of reflected light (number of photons per second) on wavelength $(\mathrm{nm})$. The instrument error was $10 \%$. The program for processing the results of measurements allowed time to accumulate readings from the instrument (photon counter) and also to conduct smoothing, scaling and elimination of incidental erroneous spikes.

\section{Recording of Fluorescence Spectra}

Cryostat sections of normal and atherosclerotic portions of aortic wall were used for study of fluorescence spectra. Specimens were prepared within 12 hours of death by layered adhesion of acetone-fixed cryostatic sections to glass. These samples were generally 120 to $130 \mu \mathrm{m}$ thick, with an area of $10 \mathrm{x}$ $15 \mathrm{~mm}$. The samples were fixed on a special object platform at an angle of $45^{\circ}$ to the direction of the beam

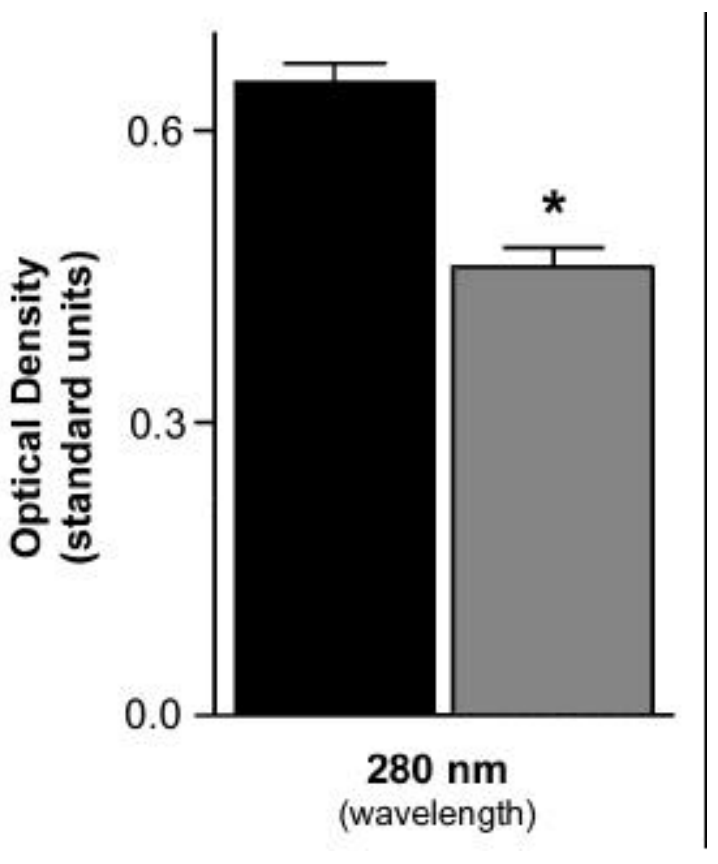

Figure 1B. Absorbance of normal aorta wall (black) and atherosclerotic plaque (grey) at $280 \mathrm{~nm}$. $* p<0.05$, Wilkoxon test. 
of incident light. The sample was excited by the lines of a DRSh-500 mercury lamp (with maximal excitation in the range of 313 and $365 \mathrm{~nm}$ ), isolated with the aid of a MUM-1 monochromator with a slit spectral width of $1 \mathrm{~nm}$. Sample luminescence was detected by an SPh-4 prism monochromator with a slit spectral width of $3 \mathrm{~nm}$ and transformed in a photoelectron multiplier (PhEU79), after which the signal entered a constant current amplifier and was registered on a chart recorder (KSP4) as a graph of the fluorescence spectrum. The graph depicted the dependence of the fluorescence intensity of the sample (standard units) on wavelength $(\mathrm{nm})$. The relative error of the prism monochromator with photoelectric recording was 5 to $10 \%$.

\section{RESULTS}

\section{Absorbance Spetra of Atherosclerotic and Normal Aortic Tissue}

Upon comparison of absorbance spectra from normal aortic tissue and from atherosclerotic plaque (without differentiation according to plaque type), two characteristic optical density maximums are observed at wavelengths 224 and $280 \mathrm{~nm}$. At the first maximum, the

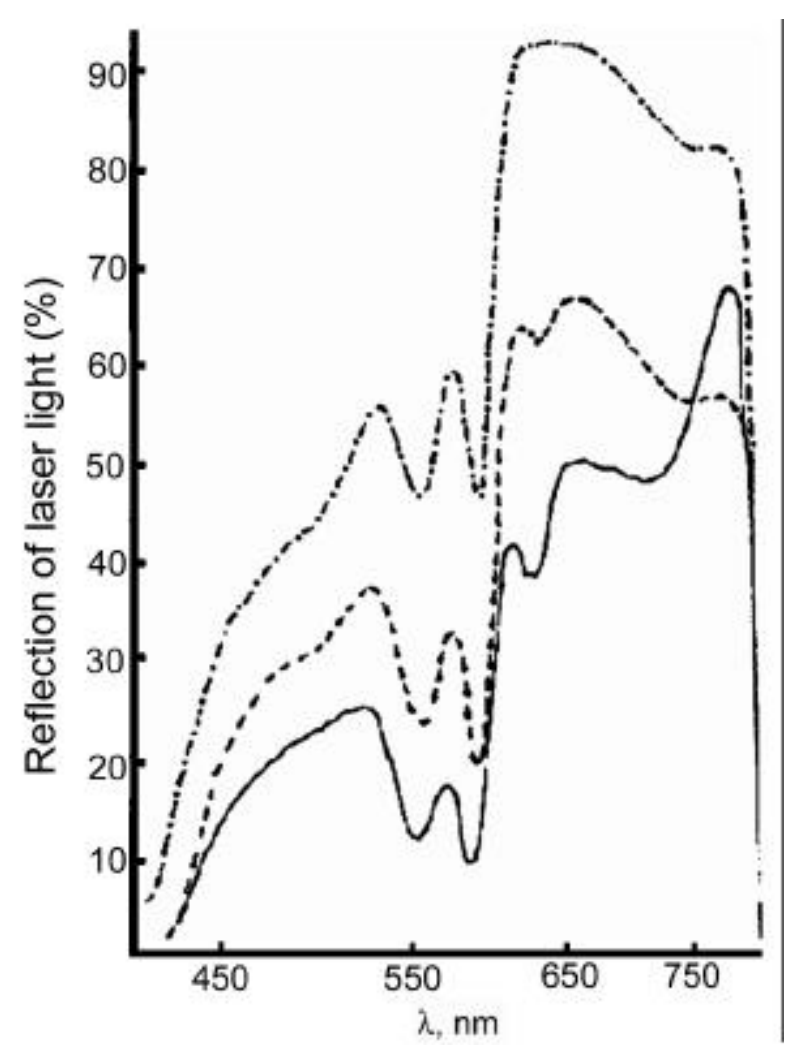

Figure 2A. The spectra of reflection in diapason of spectrum (430$760 \mathrm{~nm}$ ). Normal tissue (dashed and doted line); lipid and fibrous atherosclerotic plaques (dashed line); calcified plaque, (solid line). difference between the indices of optical density were not significant. Consequently, further analysis of light absorbance by aortic wall tissue at this wavelength was not conducted. In the first curve (Figure 1A), which corresponds to the absorbance spectrum for normal aortic wall in the ultraviolet range, the slope decreases upon approaching the second maximum, which corresponds to a wavelength of $280 \mathrm{~nm}$. This is of greatest interest. At this point, the optical density of normal tissue is $0.65 \pm 0.02$ standard units. The second curve, which corresponds to the absorbance spectrum of atherosclerotic tissue, declines more steeply and forms a maximum at the same wavelength with an optical density of $0.46 \pm 0.02$ standard units. The upward and downward slopes are approximately identical in the two absorption graphs: in the first, the absorption spectral curve declines to an optical density of $0.12 \pm 0.004$ standard units; in the second, to $0.03 \pm 0.00035$ standard units. Thus, in the ultraviolet $(280 \mathrm{~nm})$ absorption spectra of normal and atherosclerotic tissues, there is a statistically significant ( $p<0.05$; Wilkoxon test), 30\% decrease in the optical density of plaque tissue in comparison to intact tissue from aortic wall (Figure 1B).

\section{Reflection Spectra of Atherosclerotic and Normal Aortic Tissue}

In reflection spectra, it is apparent that the 520 to $770 \mathrm{~nm}$ visible wavelength range is a promising region for the exploitation of laser energy for surgical purposes (Figure 2A). Here, the difference in the reflection spectral behavior of atherosclerotic plaques and normal tissue is quite strong. The following characteristic peaks are found in the reflection spectra within the visible

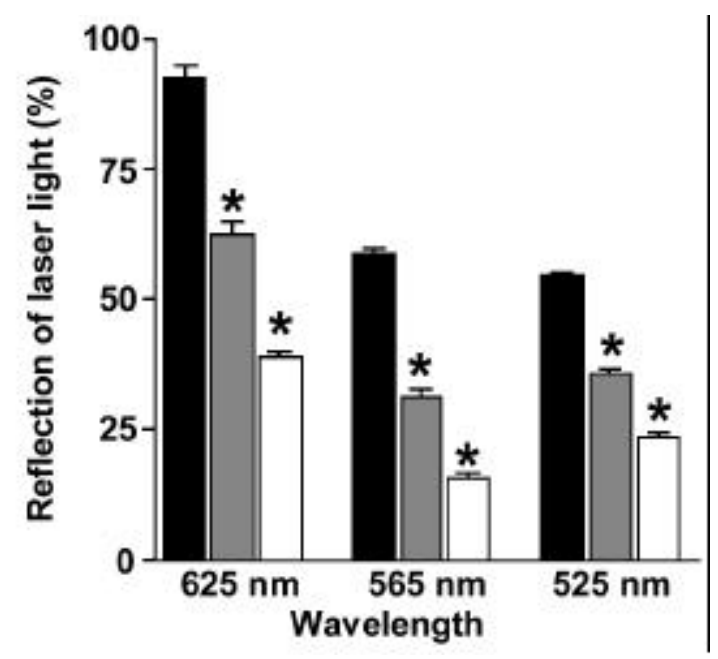

Figure 2B. Reflection values of normal (black bars), lipid and fibrous atherosclerotic plaques (grey bars), and calcified plaques (clear bars) in the visible spectral range. $* p<0.05$, Wilkoxon test. 
range. The first peak corresponds to a wavelength of $625 \mathrm{~nm}$. Here, tissue from normal aortic wall reflects, on average, $92.5 \pm 2.5 \%$ of incident light; samples with lipidic and fibrotic plaques reflect $62.5 \pm 2.5 \%$; in comparison, the coefficient of reflection for samples with calcified plaques is $39 \pm 1.0 \%$. The next reflection peak corresponds to $565 \mathrm{~nm}$. Here, normal tissue from aortic wall reflects, on average, $59.0 \pm 1.0 \%$ of incident light; lipidic and fibrotic plaques, taken as a single group, reflected $31.5 \pm 1.5 \%$; and samples with calcified plaques reflected $16.0 \pm 1.0 \%$ of incident light. The last peak lies in the vicinity of $525 \mathrm{~nm}$. As before, a pronounced reflection coefficient of $55.0 \pm 0.5 \%$ was observed for normal tissue; samples with lipidic and fibrotic plaques, considered as one group, reflect on average $36.0 \pm 1.0 \%$ of incident light; calcified plaques exhibit reflectivity of negligible intensity, namely, 24.0 $\pm 1.0 \%$ of incident light. All differences between mean reflectivity indices of these groups are statistically reliable $(p<0.05)$ (Wilkoxon test). Thus, at all points of the visible spectrum studied, the reflectivity of normal aortic wall tissue samples is significantly greater than the analogous measurement for samples with visually defined atherosclerotic lesions (Figure 2B).

\section{Fluorescence Spectra of Atherosclerotic and Normal Aortic Tissue}

The fluorescence spectrum of normal tissue is represented by a symmetric curve with a maximum

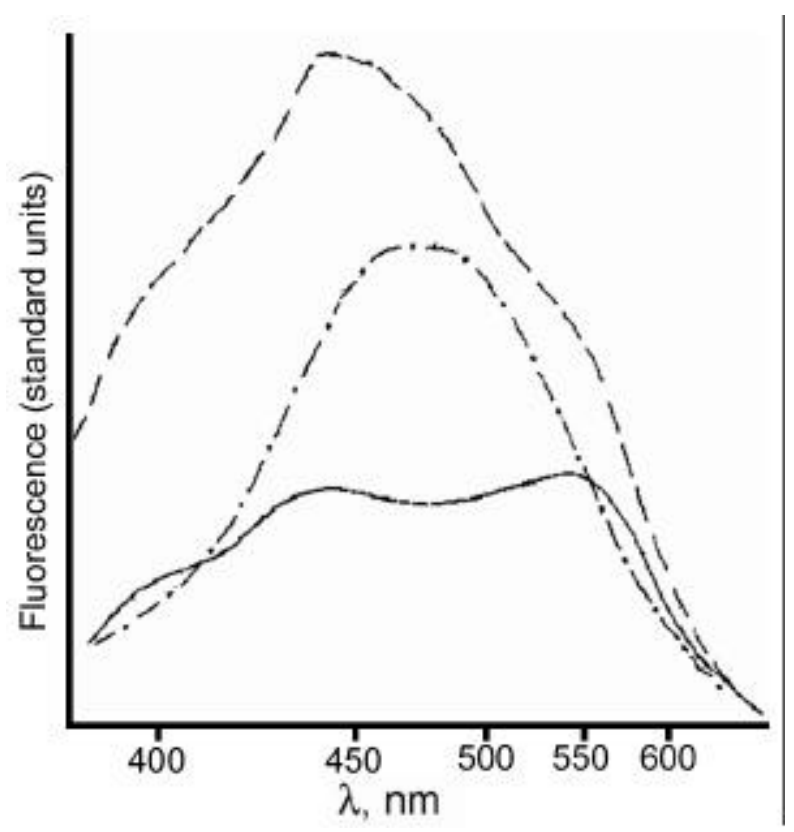

Figure 3A. The spectra of fluorescence in diapason of spectra (380$650 \mathrm{~nm}$ ). Normal tissue (dashed and doted line); lipid and fibrous atherosclerotic plaques (dashed line); calcified plaque, (solid line). fluorescence in the vicinity of $470 \pm 5 \mathrm{~nm}$. The excitation maximum in this instance lies near $365 \mathrm{~nm}$ (Figure 3A). For lipidic and fibrotic plaques, the fluorescence intensity is significantly greater than for normal tissue and the fluorescence maximum is shifted to the shorter wavelength of $440 \pm 5 \mathrm{~nm}$. The spectrum is represented by a symmetric, broadened band, the appearance of which depends on the degree of tissue damage. The fluorescence spectrum of the calcified plaque has the appearance of an asymmetric curve with two fluorescence maxima in the vicinity of 570 and $380 \mathrm{~nm}$. The latter maximum can be better distinguished by illuminating tissue with light of $313 \mathrm{~nm}$ wavelength (excitatory light).

To quantitate differences between fluorescence spectra, the ratios of fluorescence intensities for normal or atherosclerotic aortic wall tissue at optimal wavelengths (440 to $470 \mathrm{~nm}$ and 500 to $570 \mathrm{~nm}$ ) were measured at a single excitation wavelength (Figure $3 \mathrm{~B})$. For normal tissue, the 440 to $470 \mathrm{~nm}$ ratio was $0.843 \pm 0.042$, while the 500 to $570 \mathrm{~nm}$ ratio was 0.29 \pm 0.051 . At the same ratios, the corresponding indices for fibrotic and lipidic plaques were $1.19 \pm 0.035$ and $0.56 \pm 0.012$, respectively. Calcified plaques were characterized by the following indices: the 440 to $470 \mathrm{~nm}$ ratio was $0.39 \pm 0.035$ and the 500 to $570 \mathrm{~nm}$ ratio was $0.42 \pm 0.021$. All observed differences were statistically reliable to $p<0.05$ (Wilkoxon test) (Figure 3B).

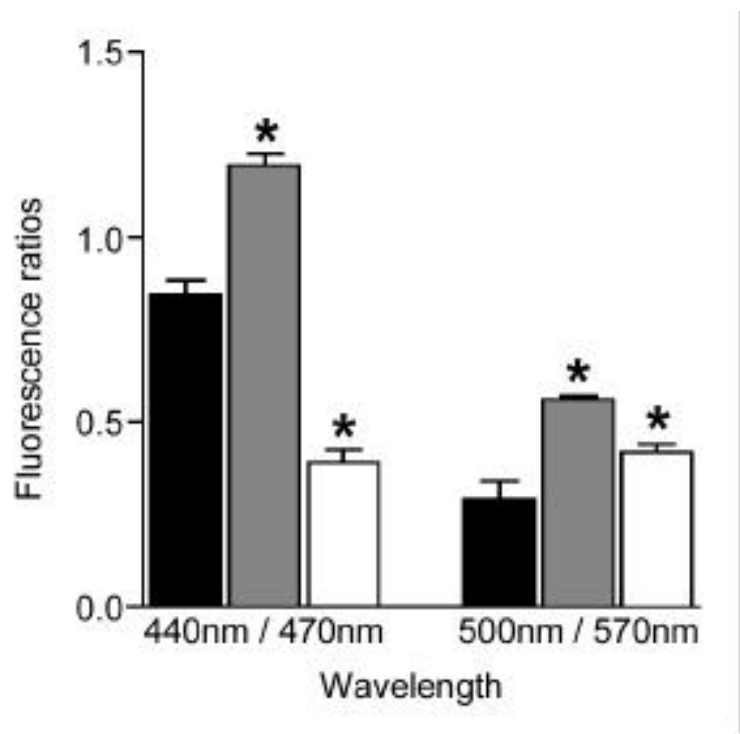

Figure 3B. Intensity of fluorescence of normal (black bars), lipid and fibrous plaques (grey bars), and calcified atherosclerotic plaques (clear bars). ${ }^{*} p<0.05$, Wilkoxon test 


\section{DISCUSSION}

The absorbance, reflection and fluorescence spectra of normal and atherosclerotic aortic wall tissue were defined. The data indicate that the visible range (principally red; $520-770 \mathrm{~nm}$ ) should be investigated further for use in laser angioplasty. In this region, on the basis of reflection and fluorescence spectra, the greatest optical differences are exhibited between intact and atherosclerotic vessel wall. The findings of these experiments are generally in agreement with other groups' findings suggesting that it is possible to distinguish between atherosclerotic lesions and healthy vessel tissue (29-33).

The manifestation of spectral differences between normal and atherosclerotic aortic wall is dependent upon: (i) infiltration of vessel wall by lipids, cholesterol and its ethers, which lower the optical density of the plaque and lead to a lowering of the absorption capacity of the tissue; (ii) the density of the tissue under examination, such that the higher the density (calcinosis), less light is reflected and, conversely, the lower the density (normal tissue), more light is reflected; and (iii) the degree of infiltration of the vessel wall by substances possessing a high capacity for luminescence (lipids) (34).

The data obtained in the current experiments are consistent with these attributes. Upon comparison of the spectral characteristics of various types of atherosclerotic plaques and normal aortic wall tissue, statistically reliable distinctions were exhibited in the following spectra. In absorption spectra in the ultraviolet range $(280 \mathrm{~nm})$, there is an average lowering of $30 \%$ in the optical density of plaque tissue, in comparison to intact aortic wall tissue. In reflection spectra the intensity of reflection for samples with lipidic and fibrotic plaques was $30-50 \%$ lower than normal aortic wall tissue at all points of the visible spectrum that were examined $(625,565$ and $525 \mathrm{~nm})$; calcified plaques were 60 - 75\% lower. In fluorescence spectra the ratio of fluorescence intensity at wavelengths 440 and 470nm (J440/470) may serve as a diagnostic parameter. If J440/470 slightly less than one, this is an indicator of normal aortic wall tissue; however, if J440/470 greater than one, it likely indicates the presence of fibrotic and lipidic plaques. In the event that $\mathrm{J} 440 / 470$ is equal to one, the possibility of transition from normal tissue to plaque must be considered. Finally, a J440/470 much less than one is likely to represent a calcified plaque.

The spectral characteristics obtained in these experiments are interpreted in terms of the basic stages of atherosclerosis, which have variable molecular composition (i.e., the relative levels of different molecules such as lipids and mucopolysaccharides). In this regard, it may be proposed that lipids and mucopolysaccharides, infiltrating the vessel wall in the stage of lipidosis and liposclerosis, lower the optical density of the tissue and absorb light less than normal (see above). This was the cause of the characteristic changes observed in absorption spectra recorded from normal aortic wall tissue and atherosclerotic tissue (lipidic and fibrotic plaques) (Figure 1).

The levels of light reflection from the surfaces of normal and atherosclerotic aortic wall differ. Moreover, the higher the density of the tissue studied, the less light it reflects (see above). This conclusion is supported by the recorded reflection spectra: normal aortic tissue has the maximal reflection intensity, while the densest medium (calcified plaque) absorbs the major share of incident light (Figure 2).

Results obtained with the aid of such precise and informative methods as the recording of fluorescence spectra also indicate differences. Indeed, the greater the tissue content of lipid, cholesterol and its ethers, the more powerfully it will luminesce, inasmuch as these substances, according to published data, possess this property to a marked degree (see above). Keeping the foregoing in mind, one may predict that lipidic and fibrotic plaques will possess the greatest fluorescence index, while normal tissue will fluoresce to a lesser degree and calcified plaques will luminesce quite weakly. This is supported by the experimental recordings of fluorescence spectra (Figure 3).

There are various limitations of the current study that should be mentioned. All the measurements made were in cryostat sections of post-mortem cadaverous aorta. As such, before more definite claims to clinical relevance can be made, it will be necessary to confirm these findings using an in vivo model. Nevertheless, in vitro measurements of thermal laser induced damage have proved to be good predictors of in vivo damage (9). Another potential problem is that the detection of subtle features such as slopes of the spectral curves or changes in fluorescence intensity may not be reliable in practice in the clinical setting. However, improvement in laser technology will likely make these issues less of a concern. A final potential limitation is that the measurements were taken from atherosclerotic and normal aortic tissue. As laser angioplasty is typically envisioned for ablation of coronary artery lesions, it will be important to confirm these data in these tissues.

The ultimate goal of this and other studies is to find a simple and safe operational system for the diagnosis of plaque type and the definition of the boundaries of the atherosclerotic lesion; these measurements are of the greatest importance for direction and delivery of intravascular laser energy. Ideally, clinical laser angioplasty should be conducted with a scanning 
protocol (to determine the plaque type and to recognize healthy vessel wall), permitting the laser wavelength to be periodically corrected with respect to spectral properties and the relief of the illuminated plaque surface. This should optimize ablation and minimize damage to healthy vessel tissue by allowing adjustment of laser energy according to lesion type.

In summary, this work has evaluated various spectral characteristics of atherosclerotic vs. normal aortic tissue and finds wavelengths where greater differences exist between atherosclerotic and normal tissue. Thus, these wavelengths should be further investigated. Despite the limitations discussed above, the current set of experiments provide the groundwork for a more complete understanding of laser-tissue interactions that may eventually result in an improvement of clinical technique.

\section{ACKNOWLEDGMENTS}

The author wishes to thank Dr. Perevod S. Desiderio (Johns Hopkins University, Baltimore, USA) for assistance with translation of the paper from Russian to English. The following Professors are thanked for their scientific tutorship and for financial support of the research: Professor V.M. Shipoulin (Head of the Department of Cardiovascular Surgery, Institute of Cardiology, Tomsk, Russia) Professor I.V. Suchodolo (Head of the Department of Histology, Siberian Medical University, Tomsk, Russia), Professor E.P. Gordov (The Institute of Atmospheric Optics), Professor M.M. Makogon (The Institute of Atmospheric Optics) and Professor S.V. Melchenko (The Institute of Atmospheric Optics).

\section{REFERENCES}

1. Libby P. Atherosclerosis. In: Fauci AS et al., eds. Harrison's Principles of Internal Medicine, 14th Edition. New York: McGraw-Hill; 1998; 1345-1352.

2. Sanborn TA. Laser angioplasty: peripheral and coronary applications. Cardiovascular Clinics. 19:181-95; 1988.

3. Wollenek G, Laufer G, and Haschkovitz H. Koronare laserantioplastie. Herz 10: 351-356; 1985.

4. Abela GS, Suger JM, Barbieri E, et al. Laser angioplasty with angioscopic guidance in humans. Journal of the American College of Cardiology 8: 184-192; 1986.

5. Litvack F, Grundfest WS, Segalowiatz J, et al. Interventional cardiovascular therapy by laser and thermal angioplasty. Circulation. 81 (Supplement 4):109-116; 1990.

6. Abela GS, Normann S, Cohen D, et al. Effects of carbon dioxide, Nd-YAG and argon laser radiation or coronary atheromatous plaques. American Journal of Cardiology 50: 1199-1205; 1982.

7. Ginsburg R, Kim DS, Guthaner D, et al. Salvage of an ischemic limb by laser angioplasty: description of a new technique. Clinical Cardiology 7: 54-8; 1984.

8. Geschwind H, Boussignac G, Teisseire B, et al. Percutaneous transluminal laser angioplasty in man. Lancet. 1(8381): 844;
1984.

9. Isner JM, Rosenfield $\mathrm{K}$, White $\mathrm{CJ}$, et al. In vivo assessment of vascular pathology resulting from laser irradiation. Analysis of 23 patients studied by directional atherectomy immediately after laser angioplasty. Circulation 85: 2185-2196; 1992.

10. Litvack F, Eigler NL and Forrester JS. Excimer laser coronary angioplasty. Science and Medicine 3(1): 42-51; 1996.

11. Grundfest WS, Litvack F, Forrester JS, et al. Laser ablation of human atherosclerotic plaque without adjacent tissue injury. Journal of the American College of Cardiology 5:929-933; 1985.

12. Isner JM, Donaldson RF, Deckelbaum LI, et al. The excimer laser: gross, light microscopic and ultrastructural analysis of potential advantages for use in laser therapy of cardiovascular disease. Journal of the American College of Cardiology 6:1102$1109 ; 1985$.

13. Sartori M, Henry PD, Sauerbrey R, et al. Tissue interactions and measurement of ablation rates with ultraviolet and visible lasers in canine and human arteries. Lasers in Surgery and Medicine 7:300-306; 1987.

14. Bittl JA. Excimer laser coronary angioplasty: relative risk analysis of acute and follow-up results in 200 patients. Circulation 86: 71-80; 1992.

15. Bittl JA, Sanborn TA, Tcheng JE, et al. Clinical success, complications and restenosis rates with excimer laser coronary angioplasty, the percutaneous excimer laser coronary angioplasty registry. American Journal of Cardiology 70:15331539; 1992.

16. Margolis JR, Metha SM. Excimer laser coronary angioplasty. American Journal of Cardiology 69: 3-11F; 1992.

17. Cook SL, Eigler NL, Shefer A, et al. Percutaneous excimer laser coronary angioplasty of lesions not ideal for balloon angioplasty. Circulation 84:632-643; 1991.

18. Bittl JA, Sanborn TA. Excimer laser-facilitated coronary angioplasty. Relative risk analysis of acute and follow-up results in 200 patients. Circulation 86:71-80; 1992.

19. Holmes DR, Vliestra RE, Smith HC, et al. Chronic total obstruction and short-term outcome: the excimer laser coronary angioplasty registry experience. Mayo Clinics Proceedings 68: 5-10; 1993.

20. Lee G. Eximer laser coronary angioplasty: it's time for a critical evaluation. American Journal of Cardiology 69:1640-1643; 1992.

21. Margolis JR, Litvack F, Krauthamer D, et al. Coronary angioplasty with laser and high frequency energy. eximer laser coronary angioplasty: American multicenter experience. Herz 15: 223-232; 1990.

22. Bittl JA. Clinical results with excimer laser coronary angioplasty. Seminars in Interventional Cardiology 1: 129-134; 1996.

23. Holmes DR Jr, Mehta S, George CJ, et al. Excimer laser coronary angioplasty: the new approaches to coronary intervention (NACI) experience. American Journal of Cardiology 80: 99K-105K.

24. Strikwerda S, Koolen JJ, de Feyter PJ, et al. Excimer laser coronary angioplasty in the Netherlands: preamble for a randomized study. American Heart Journal 125: 838-847; 1993.

25. Appelman YEA, Piek JJ, Strikwerda S, et al. Randomised trial of excimer laser angioplasty versus balloon angioplasty for treatment of obstructive coronary artery disease. Lancet 347:79$84 ; 1996$.

26. Appelman, YEA; Piek, JJ; Redekop, WK, et al. Clinical events following excimer laser angioplasty or balloon angioplasty for complex coronary lesions: subanalysis of a randomised trial. 
Heart 79: 34-38; 1998

27. van Leeuwen TG, Velema E, Pasterkamp G et al. Saline flush during excimer laser angioplasty: short and long term effects in the rabbit femoral artery. Lasers in Surgery and Medicine 23: 128-140; 1998.

28. Hasse KK, Rose C, Duda S et al. Perspective of coronary excimer laser angioplasty: multiplexing, saline flushing and acoustic ablation controls. Lasers in Surgery and Medicine 21: 72-78; 1997.

29. Leon MB, Lu DY, Prevosti LG, et al. Human arterial surface fluorescence: atherosclerotic plaque identification and effects of laser atheroma ablation. Journal of the American College of Cardiology 12: 94-102; 1988.

30. Arakawa K, Papazoglou T, Papaioannou T, et al. XeCl excimer laser-induced fluorescence for selective ablation of atheromatous tissue. Japanese Circulation Journal 55: 10941095; 1991.

31. Cutruzzola FW, Stetz ML, O'Brien KM, et al. Change in laserinduced arterial fluorescence during ablation of atherosclerotic plaque. Lasers in Surgery and Medicine 9:109-116; 1989.

32. Morguet AJ, Korber B, Abel B, et al. Autofluorescence spectroscopy using a $\mathrm{XeCl}$ excimer laser system for simultaneous plaque ablation and fluorescence excitation. Lasers in Surgery and Medicine 14: 238-248; 1994.

33. Deckelbaum LI, Lam JK, Cabin HS, et al. Discrimination of normal and atherosclerotic aorta by laser-induced fluorescence. Lasers in Surgery and Medicine 7:330-335; 1987.

34. Kats VA, Vorob'ev SV, Stebakova LN, et al. Aspects of morphology of photodynamic destruction. Arkhiv Patologi (Moskva) 58: 1-68; 1996.

Nikolay Korovin graduated from the Medico-Biological Faculty and the Treatment Faculty of the Siberian Medical University (Tomsk, Russia) in 1997 and 1998, respectively. He is currently a clinical intern in the Department of Cardiovascular Surgery, Institute of Cardiology (Tomsk, Russia). He is also pursuing post-graduate studies in the Department of Anatomy and General Pathology, Siberian Medical University. His research concerning the spectral characteristics of normal and atherosclerotic aortic tissue was begun in 1992 at the Department of Anatomy and General Pathology. 